\title{
A comprehensive study on the role of the Yersinia pestis virulence markers in an animal model of pneumonic plague
}

\author{
W. E. Kaman • S. Hawkey • D. van der Kleij • \\ M. P. Broekhuijsen • N. J. Silman • F. J. Bikker
}

Received: 19 November 2010 / Accepted: 4 January 2011 /Published online: 6 April 2011

(C) The Author(s) 2011. This article is published with open access at Springerlink.com

\begin{abstract}
We determined the role of Yersinia pestis virulence markers in an animal model of pneumonic plague. Eleven strains of $Y$. pestis were characterized using PCR assays to detect the presence of known virulence genes both encoded by the three plasmids as well as chromosomal markers. The virulence of all $Y$. pestis strains was compared in a mouse model for pneumonic plague.
\end{abstract}

W. E. Kaman · D. van der Kleij · M. P. Broekhuijsen · F. J. Bikker

TNO Defence, Security and Safety,

2280 AA Rijswijk, the Netherlands

W. E. Kaman

Department of Medical Microbiology and Infectious Diseases,

Erasmus MC,

3015 Rotterdam, the Netherlands

S. Hawkey $\cdot$ N. J. Silman

Research Department,

Centre for Emergency Preparedness and Response,

Health Protection Agency,

Porton Down,

SP4 0JG Salisbury, UK

W. E. Kaman • F. J. Bikker

Department of Oral Biochemistry,

Academic Centre for Dentistry Amsterdam,

University of Amsterdam,

Amsterdam, the Netherlands

W. E. Kaman • F. J. Bikker

VU University Amsterdam,

1081LA Amsterdam, the Netherlands

W. E. Kaman $(\square)$

Department of Medical Microbiology and Infectious Diseases,

Erasmus MC,

's-Gravendijkwal 230,

3015 Rotterdam, the Netherlands

e-mail: w.kaman@erasmusmc.nl
The presence of all known virulence genes correlated completely with virulence in the Balb/c mouse model. Strains which lacked HmsF initially exhibited visible signs of disease whereas all other strains (except wild-type strains) did not exhibit any disease signs. Forty-eight hours post-infection, mice which had received $\mathrm{HmsF}^{-}$strains regained body mass and were able to control infection; those infected with strains possessing a full complement of virulence genes suffered from fatal disease. The bacterial loads observed in the lung and other tissues reflected the observed clinical signs as did the cytokine changes measured in these animals. We can conclude that all known virulence genes are required for the establishment of pneumonic plague in mammalian animal models, the role of $\mathrm{HmsF}$ being of particular importance in disease progression.

Yersinia pestis, a Gram-negative bacillus is the causative agent of the disease plague which is a zoonotic disease primarily affecting rodents; the human host plays no part in the long term survival of $Y$. pestis. Transmission between rodents occurs via fleas, $Y$. pestis being acquired from an infected blood meal, infection in these hosts being confined to the alimentary canal. Transfer to other rodents occurs via regurgitation of the bacteria as the fleas repeatedly try to feed, a consequence of infection by $Y$. pestis is the blockage of the proventricular valve thus preventing blood meals entering the stomach (Perry and Fetherston 1997). The human disease manifests itself in three different forms; bubonic and primary scepticemic plague both of which are spread by fleas, although the former has a fatality rate of $40-60 \%$ whereas the latter is $100 \%$ fatal if untreated. The third manifestation is primary pneumonic plague which is 
spread by aerosol droplets and, like scepticemic plague, is $100 \%$ fatal if left untreated. Secondary pneumonic plague can occur in cases of bubonic plague that remain untreated (Prentice and Rahalison 2007).

A number of plasmid and chromosome-encoded virulence genes have been identified in $Y$. pestis including those involved in Congo red binding $\left(\mathrm{Crb}^{+}\right.$phenotype), which forms the basis of an assay which is used as an indicator of the presence of a number of virulence genes collectively involved in iron uptake, and the pigmentation phenotype $\left(\mathrm{Pgm}^{+}\right.$phenotype) encoded by a 102-kb locus (pgm locus) part of which encodes a siderophore-dependent iron transport system (Perry et al. 2004). $\mathrm{PGM}^{-}$bacteria are avirulent in the mouse model unless infection occurs via the intravenous route or bacteria are supplemented with an exogenous source of iron (Staggs et al. 1994). Other important virulence factors are the proteins pesticin, plasminogen activator (pPCP1; $9.5 \mathrm{~kb}$ ), and the $\mathrm{F} 1$ (pMT1; $110 \mathrm{~kb}$ ) and V-antigens (pCD1; $75 \mathrm{~kb})$. All of these virulence factors are plasmid encoded as indicated, and loss of any of these plasmids variably affects virulence in animal models dependent upon the route of infection (Zauberman et al. 2009). Indeed, in wild-type strains, growth at $25^{\circ} \mathrm{C}$ compared with $37^{\circ} \mathrm{C}$ was found to significantly increase the $\mathrm{LD}_{50}$ when mice were infected via the aerosol route, however, other routes of infection were unaffected (Perry and Fetherston 1997). The kinetics of pneumonic plague development have recently been characterized and described in both the mouse as well as in the brown Norwegian rat model (Agar et al. 2008; Anderson et al. 2009; Agar et al. 2009). In these model systems, the fully virulent strain $\mathrm{CO} 92$ was used as the challenge strain and similar $\mathrm{LD}_{50}$ 's as well as times to death were observed in both animal models. In these models, it was postulated that the observed early pro-inflammatory response was induced by the type III secretion system (encoded by the pCD1 plasmid) and its associated effectors, whilst the latter pro-inflammatory response resulted from the production of a number of cytokines and chemokines, specifically IL- $1 \beta$, IL- $1 \alpha$, IFN- $\gamma$, IL-12, and IL-6 (Agar et al. 2008).

There are numerous strains of $Y$. pestis residing in culture collections throughout the world, many of which remain uncharacterized in terms of their virulence in pneumonic and bubonic plague animal models. Many molecular assays for detecting the presence of genes encoding the known key virulence determinants have also been described (Tomaso et al. 2008; Matero et al. 2009). However, although these assays exist and have been used for clinical diagnosis or environmental detection there are no reports correlating the presence of known virulence gene markers as detected by polymerase chain reaction (PCR) with virulence in animal models of pneumonic plague. This study, therefore, characterized the strains of $Y$. pestis which were available from the Health Protection Agencies National Collection of Type Cultures (NCTC) in London for the presence of known virulence gene markers and correlated their virulence in the mouse model of pneumonic plague.

\section{Material and methods}

Culture of bacterial strains for molecular analysis Y. pestis strains (see Table 2) were obtained as lyophilized cultures directly from the National Collection of Type Cultures (NCTC), HPA (London, UK). Strains were reconstituted in $0.5-\mathrm{mL}$ sterile water, aliquots plated onto Columbia Horse Blood agar plates (bioMérieux) and incubated at $37^{\circ} \mathrm{C}$ for $48 \mathrm{~h}$. Cultures were checked for purity before subculture onto fresh Columbia Horse Blood agar and CMOX agar. Single colonies (six) were examined for each of the strains from both media types by polymerase chain reaction (PCR) assay (see below). Cultures grown on the previously described media were also incubated at $28^{\circ} \mathrm{C}$ and colonies examined using the same methodology (Tomaso et al. 2008).

Culture of bacterial strains for challenge stocks To prepare challenge stocks of $Y$. pestis, bacteria were cultured in Brain Heart Infusion (BHI) broth for $24 \mathrm{~h}$ at $26^{\circ} \mathrm{C}$. The bacterial pellet was washed twice in sterile phosphate-buffered saline (PBS), resuspended in the same buffer and stored overnight at $4^{\circ} \mathrm{C}$. The concentration was determined by plating 10 fold serial dilutions on Trypticase Soy Agar (TSA) plates. Plates were incubated at $26^{\circ} \mathrm{C}$, and $\mathrm{CFU}$ enumerated after a 3-day incubation. Pulmonary infection with $Y$. pestis was performed by intranasal (i.n.) inoculation of $2 \times 10^{5} \mathrm{CFU}$ bacteria in a $50-\mu \mathrm{L}$ volume. Five mice were used for each $Y$. pestis strain, with another five mice used as control (noninfected). Three days post-infection mice were euthanized by an overdose of Nembutal, and the bacterial load within the organs was determined.

Polymerase chain reaction Template material for PCR was produced by thermal lysis of emulsified colonies obtained as described previously (Tomaso et al. 2008). Used primer and probe sets are listed in Table 1. Colonies were emulsified in sterile distilled water $(100 \mu \mathrm{L})$ and heated at $100^{\circ} \mathrm{C}$ for $10 \mathrm{~min}$. Aliquots were examined for sterility by plating onto Columbia Horse Blood agar before thermal lysates were removed from containment facilities. PCR reactions contained: $2 \times$ Fast Universal Master mix (Applied Biosystems $) 10 \mu \mathrm{L}$, forward primer $(18 \mathrm{nmol} / \mathrm{L}) 1 \mu \mathrm{L}$, reverse primer $(18 \mathrm{nmol} / \mathrm{L}, 1 \mu \mathrm{L})$; probe $(5 \mathrm{mmol} / \mathrm{L}) 1 \mu \mathrm{L}$, RNase free water $5 \mu \mathrm{L}$, DNA template $2 \mu \mathrm{L}$. Reactions 
Table 1 PCR primers, probes, and loci used

\begin{tabular}{lllll}
\hline Plasmid & Locus & \multicolumn{2}{l}{ Sequences, $3^{\prime} \rightarrow 5^{\prime}$} & \\
\cline { 3 - 5 } & & Forward primer & Reverse primer & Probe \\
\hline pCD1 & lcrV & CGG CGG TTA AAG AGA AAT GC & CAT CGC CGA ATA CAC AAT GG & TAC TGC CAT GAA CGC C \\
pMT1 & $c a F 1$ & TTG GCG GCT ATA AAA CAGG AA & CAC CCG CGG CAT CTG TA & CAC TAG CAC ATC TGT TAA C \\
pPCP1 & $p s t 1$ & CGG CAA TCG TTC CCT CAA & GGT CAG GAA AAA GAC GGT GTG A AAC CAT GAC ACG GTA GAC T \\
HmsF $^{\mathrm{a}}$ & $h m s H F R S$ & CGG AGA AGC CAA CGT TCG T & TCT TTC ACT TTG CGG CAA TG & CCG CCT GCA CAA CG \\
\hline
\end{tabular}

${ }^{\mathrm{a}}$ Chromosomal

were submitted to 40 cycles ( $A B I 7500$ fast Protocol) comprising $95^{\circ} \mathrm{C}, 3 \mathrm{~s} ; 60^{\circ} \mathrm{C}, 30 \mathrm{~s}$.

Animals Balb/c mice (Harlan, the Netherlands) were used. Adult (6- to 8-week-old) animals were kept in sterile isolators in a biohazard animal unit. Animals were fed irradiated food (Harlan) and allowed to imbibe acidified water ad libitum. The infected mice were monitored regularly for clinical status and weighed daily. All experimental procedures performed on the animals were approved by the Ethical Committee on Animal Experimentation at TNO.

Determination of bacterial load in tissues of infected animals Lungs, spleens and livers of the infected animals were aseptically removed to determine the number of bacteria in these organs. Organs were homogenized in 5$\mathrm{mL}$ sterile PBS using the MicroBiomaster (Seward, UK). The homogenates were serially diluted and plated on TSA plates. Plates were incubated at $26^{\circ} \mathrm{C}$, and CFUs were determined after a 3-day incubation.

Infection of human PBMCs in vitro Human peripheral blood mononuclear cells (PBMC) were isolated from buffy coats (Sanquin Bloodbank, Rotterdam), derived from three different volunteers, within $24 \mathrm{~h}$ after venepuncture by density centrifugation on Ficoll Paque Plus (Amersham Pharmacia). PBMCs were washed and seeded in 24-well culture plates (Costar) at a concentration of $10^{6}$ cells per well in RPMI-1640 (BioWhittaker) supplemented with $10 \%$ FCS $(P A A)$. The $Y$. pestis strains, grown in $\mathrm{BHI}$ medium at $26^{\circ} \mathrm{C}$, were used to infect the PBMCs at a multiplicity of infection of 10 . Subsequently, the PBMCs were incubated at $37^{\circ} \mathrm{C}$ in $5 \% \mathrm{CO}_{2}$ atmosphere. Levels of TNF- $\alpha$ and IL- 6 were determined in the culture supernatants by enzyme-linked immunosorbent assay (ELISA; PeliKine compact; Sanquin) at 4 and 48 h post-infection, respectively. Cytotoxicity of the $Y$. pestis strains was determined by measuring LDH activity in the culture supernatant $48 \mathrm{~h}$ post-infection using the Cytotoxicity Detection kit (Roche).
Statistical analysis Data were analyzed for statistical significance using a two-way ANOVA for the weight curve and unpaired, one-tailed Students $t$ tests for bacterial load, cytokine levels and cytotoxicity. All data are presented as mean \pm standard error of the mean.

\section{Results}

Molecular analysis of Y. pestis strains Currently, the link between the presence of different gene markers and virulence of $Y$. pestis isolates is rather unclear; the absolute determination of virulence requires an in vivo challenge study to be performed. Moreover, the culture collections in different countries, containing these pathogens often do not have access to such in vivo study data. We therefore designed PCR primers and probes against the known plasmid-derived virulence marker genes $L c r V$, pst 1 , and $c a F 1$, as well as the chromosomal locus $\operatorname{HmsF}$ (Table 1). The presence or absence of the different genetic markers was determined using real-time PCR, based upon amplification of the gene rather than detection of the mRNA (Table 2). Growth on CMOX agar plates also allowed the ability of the strains to adsorb the dye Congo red to be evaluated. For each strain there was complete correlation of the phenotypic property of Congo red adsorption with the presence of the HmsF locus, as expected. Only NCTC strains 570, 5923, and 8779 did not show the classical redpigmented colonies when grown on CMOX agar. Pigmentation of the colonies was observed in $\mathrm{HmsF}^{+}$strains irrespective of the growth temperature or whether strains had been previously sub-cultured on alternative growth media.

Assessment of $Y$. pestis virulence in a mouse model of pneumonic plague To determine the importance of the role of the different $Y$. pestis virulence markers examined by PCR (HmsF, pCD1, and pMT1) in the progression of pneumonic plague, mice were intranasally infected with the $Y$. pestis strains (Table 2). After $24 \mathrm{~h}$, the mice that received the $Y$. pestis strains lacking HmsF, had a ruffled fur and 
Table 2 Yersinia pestis strains used, including Ct values from the real-time PCR analysis

\begin{tabular}{|c|c|c|c|c|c|}
\hline \multirow[t]{2}{*}{ NCTC number } & \multirow[t]{2}{*}{ Designation } & \multicolumn{4}{|l|}{$\mathrm{Ct}$ values } \\
\hline & & $\mathrm{HmsF}$ & pMT1 & pCD1 & pPCP1 \\
\hline 10030 & Human, fatal bubonic plague & $19.05(+)$ & $19.40(+)$ & $17.67(+)$ & $24.85(+)$ \\
\hline 10329 & Isolated Nairobi 1963 & $19.47(+)$ & $19.76(+)$ & $18.01(+)$ & $28.50(+)$ \\
\hline 10029 & Human, bubonic plague & $23.41(+)$ & $23.96(+)$ & $20.26(+)$ & $20.13(+)$ \\
\hline 10330 & Isolated Nairobi 1963 & $19.20(+)$ & $19.83(+)$ & $17.84(+)$ & $17.07(+)$ \\
\hline 8775 & Isolated Manchester 1953, ex India & $19.83(+)$ & $20.73(+)$ & $18.83(+)$ & $17.97(+)$ \\
\hline 570 & Human, fatal bubonic plague, Bombay 1920 & n.d. $(-)$ & $22.10(+)$ & $19.78(+)$ & $28.91(+)$ \\
\hline 5923 & Java type strain 1939 & n.d. $(-)$ & $22.10(+)$ & $19.36(+)$ & $17.60(+)$ \\
\hline 2868 & Isolated Bombay 1928 & $20.74(+)$ & $21.54(+)$ & n.d. $(-)$ & $22.52(+)$ \\
\hline 5924 & Isolated from Javan strain 1939 & $18.79(+)$ & $19.62(+)$ & n.d. $(-)$ & $17.93(+)$ \\
\hline 2028 & Javan plague, Java 1925 & n.d. $(-)$ & $23.71(+)$ & n.d. $(-)$ & $18.73(+)$ \\
\hline 8779 & Isolated Manchester 1953 & n.d. $(-)$ & n.d. $(-)$ & n.d. $(-)$ & $18.50(+)$ \\
\hline
\end{tabular}

n.d. not detected

showed a slight decrease in body weight (Fig. 1). All other mice looked healthy and were behaving normally, with no visible symptoms of disease. After $48 \mathrm{~h}$ upon infection, the mice infected with $\mathrm{HmsF}^{-}$strains apparently were able to control the infection and gained body mass. However, the mice infected with the wild-type (i.e., positive for all tested markers) Y. pestis strains started to show a significant loss of body mass (Fig. 1) and appeared listless and hunched up. At $72 \mathrm{~h}$ post-infection, the mice that received the wild-type $Y$. pestis started to lose even more body mass and seemed totally unable to control the infection, whereas all mice infected with the mutant $Y$. pestis strains were behaving normally and showed no clinical signs of pneumonic plague.

Post-mortem analysis of tissues To investigate whether the bacterial clearance differed when one or more of the virulence markers were lacking, the bacterial loads in

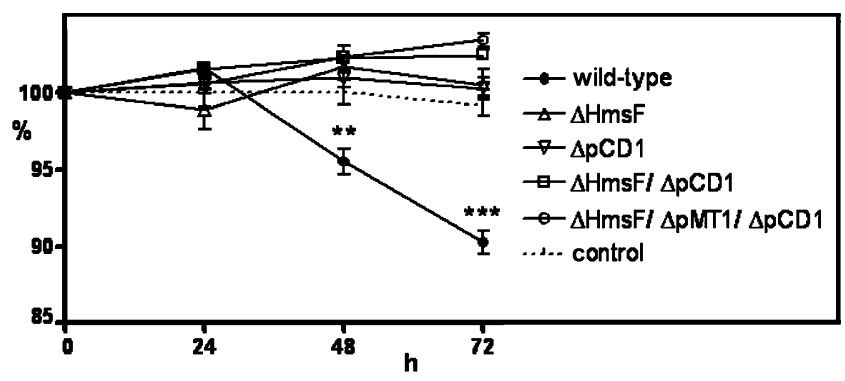

Fig. 1 Mice infected with the mutant Yersinia pestis strains show no decrease (in percent) in body mass (in percent). Balb/c mice were i.n. infected with $2 \times 10^{5} \mathrm{CFU}$ wild-type or mutant $Y$. pestis and monitored for body mass. Results are expressed as means \pm SEM ( $n=5$ per strain); significance was determined by two-way ANOVA $(* * p<0.01 ; * * * p<0.001)$ spleen, lung and liver were determined. In the organs of mice infected with $Y$. pestis strains containing all the investigated virulence genes (as analyzed by PCR), mean bacterial counts of approximately $10^{6} \mathrm{CFU} / \mathrm{mg}$ lung tissue were found (Fig. 2a). However, in animals challenged with strains missing one or more virulence genes, the bacterial loads in the lungs were at least 4 logs lower than was observed with the fully virulent strains. Similar results were obtained when liver (Fig. 2c) and spleen tissue (Fig. 2b) were analyzed.

In addition, the mass of lung, spleen and liver tissues was determined post-mortem. Interestingly, the masses of the lungs from mice challenged with strains possessing a full complement of virulence genes was significantly higher than from mice challenged with strains missing one or more virulence genes (Fig. 3a). This was presumably due to the observed hemorrhage in tissues from mice challenged with virulent strains (Fig. 3b). Lungs from mice infected with the mutant strains uniformly exhibited lower lung masses.

In vitro analysis of the role of the $Y$. pestis virulence markers in cytotoxicity To determine the role of the virulence plasmids in $Y$. pestis induced cytotoxicity human PBMCs were isolated from three volunteers and cultured in the presence of the $Y$. pestis strains described in Table 2 . After $48 \mathrm{~h}$, cytotoxicity was determined by measuring the release of lactate dehydrogenase in the culture supernatant due to cell lysis. As expected the wild-type strains were highly toxic to the PBMCs. In contrast, a slight decrease in cytotoxicity was observed in the $Y$. pestis strains lacking pCD1 compared with the wild-type strains (Fig. 4), which is probably due to the absence of the TTSS that is encoded by the pCD1 plasmid. In the $Y$. pestis strains lacking the 
Fig. 2 Decreased bacterial loads $(\log \mathrm{CFU} / \mathrm{mL})$ in case one or more of the virulence markers is missing. Balb/c mice were i.n. infected with $2 \times 10^{5} \mathrm{CFU}$ wild-type (wt) or mutant Yersinia pestis $(F \Delta \mathrm{HmsF}, 1 \Delta \mathrm{pCD} 1, F 1$ $\Delta \mathrm{HmsF} / \Delta \mathrm{pCD} 1, F 12, \Delta \mathrm{HmsF} /$ $\Delta \mathrm{pCD} 1 / \Delta \mathrm{pMT} 1, C$ control) and euthanized at $72 \mathrm{~h}$ post-infection. Numbers of CFU per organ in lung (a), spleen (b), and liver (c) were determined. Results are expressed as means \pm SEM $(n=5$ per strain); significance was determined by comparison of the mutant strains to the wild-type strain using Student's $t$ test analysis $\left({ }^{*} p<0.05 ; * * p<0.01\right.$ $* * * p<0.001$ )
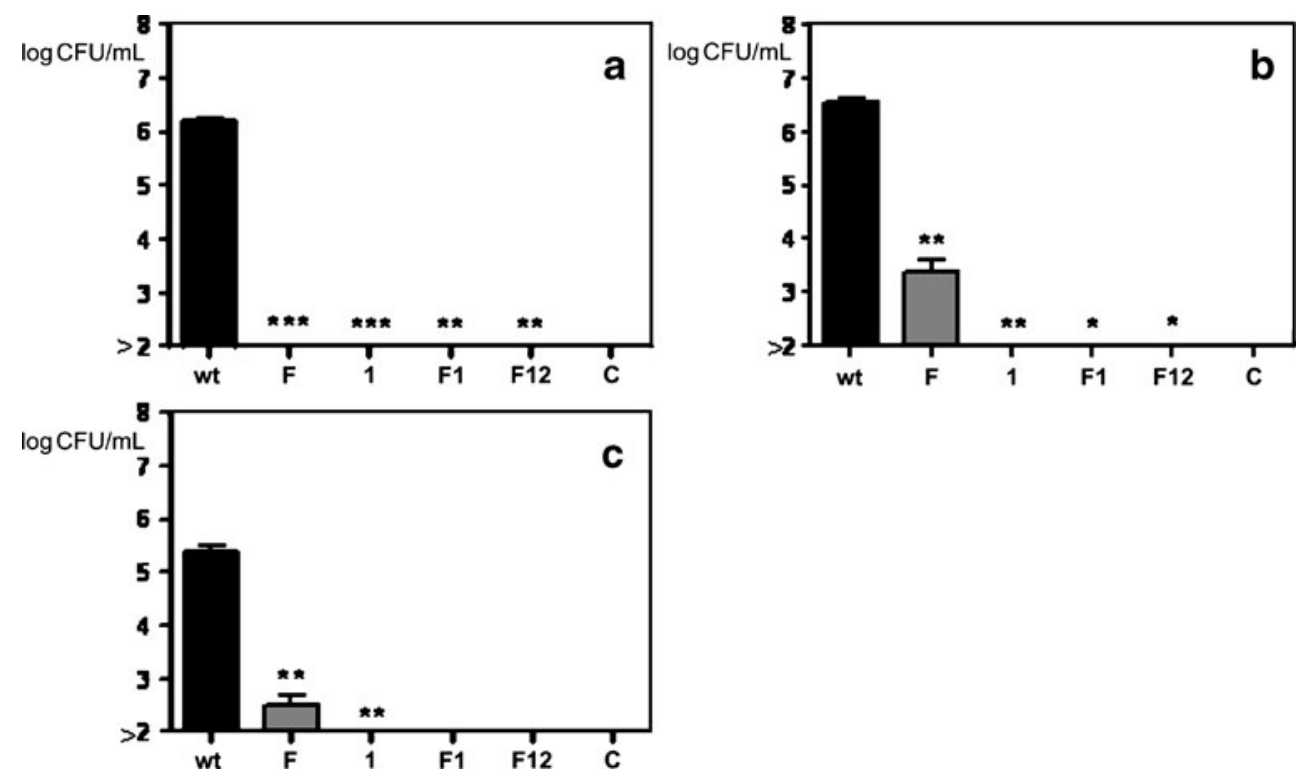

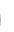

\section{Discussion}

HmsF but possessing pCD1, no decrease in cytotoxicity was detected.

In vitro analysis of $Y$. pestis induced cytokine production The production of pro- and anti-inflammatory cytokines by immunomodulatory cells is a crucial component of the host response to control bacterial infection. To study the role of the $Y$. pestis virulence plasmids in the induction of cytokine production, human PBMCs were isolated from three volunteers and cultured in the presence of the $Y$. pestis strains described in Table 2. At 4 and $48 \mathrm{~h}$ post-infection, levels of TNF- $\alpha$ and IL- 6 were measured in the culture supernatants using ELISA. A significant increase in cytokine production was observed in all $\mathrm{pCD}^{-}$strains (Fig. 5). The only exception was the $Y$. pestis strain negative for HmsF, pCD1, and pMT1, which showed an increase in IL-6 levels, but no increase in TNF- $\alpha$ production. Again, no differences were observed between the wild-type strains and the $\mathrm{HmsF}^{-}$strains.

This study set out to determine the relationship between the presence of a number of key $Y$. pestis genes and virulence as determined by the mouse model of pneumonic plague. The aim was to be able to use a simple molecular assay in order to predict virulence and hence outcome in the mouse model.

The binding by $Y$. pestis strains of the dye Congo red has been historically used as an indicator of the presence of a number of virulence traits related to iron uptake (Perry et al. 1990). The combination of the virulence factors collectively known as the $\mathrm{Pgm}^{+}$(pigmentation) phenotype is used to describe the genetic linkage between the hmsHFRS locus required for Congo red binding $\left(\mathrm{Crb}^{+}\right)$and the virulence genes encoding a siderophore-based iron-uptake system (Perry et al. 1990); loss of this locus from Y. pestis occurs at high frequency (Brubaker 1969) and is the result of a $102 \mathrm{~kb}$ chromosomal deletion (Hare and McDonough

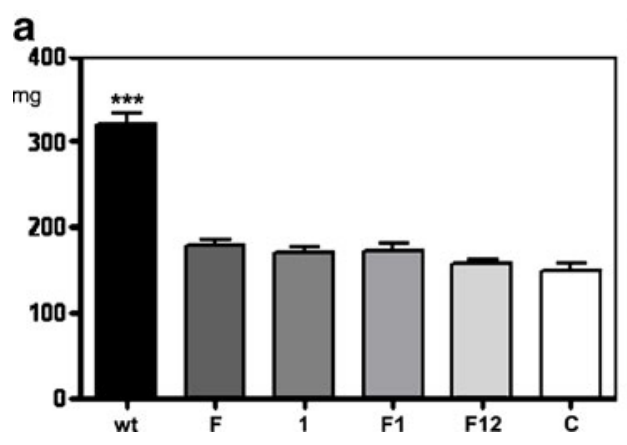

Fig. 3 Infection with wild-type Yersinia pestis leads to increased lung mass (milligrams) and hemorrhage in the tissues. Balb/c mice were i.n. infected with $2 \times 10^{5} \mathrm{CFU}$ wild-type or mutant $Y$. pestis. At $72 \mathrm{~h}$ postinfection the animals were euthanized and lung mass was determined (a). An example of hemorrhage in the tissues is shown in (B). Results b
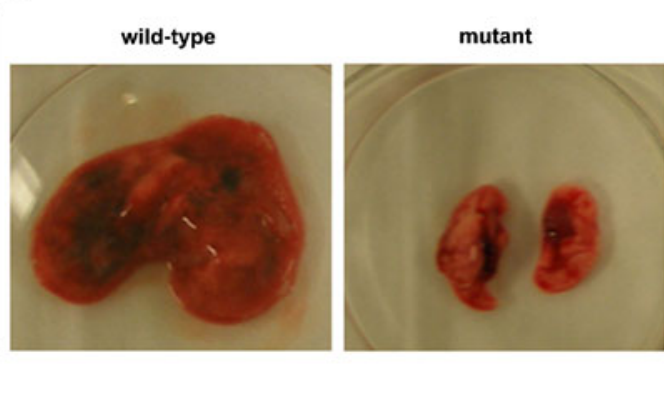

are expressed as means \pm SEM ( $n=5$ per strain). Significance was determined by comparison of the lung masses of the infected animals to the uninfected, control animals using Student's $t$ test analysis $(* * * p<$ 0.001); for abbreviations of strains, see Fig. 2 


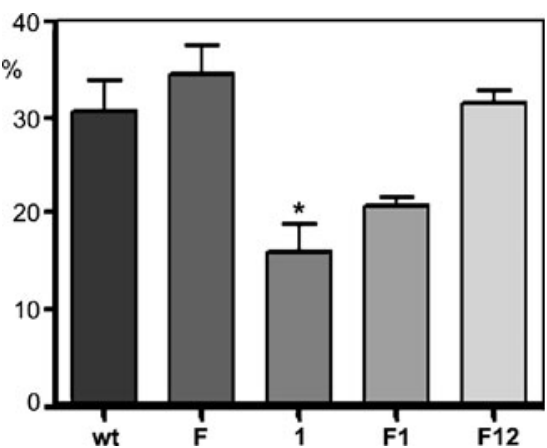

Fig. 4 Absence of the pCD1 plasmid leads to a slight decrease in cytotoxicity (in percent). Human PBMCs were infected with wild-type or mutant Yersinia pestis at a multiplicity of infection of 10 . At $48 \mathrm{~h}$ post-infection the cytotoxicity was determined by measuring the lactate dehydrogenase activity. Data are representative of three independent experiments; results are expressed as means \pm SEM. Significance was determined by comparison of the mutant strains to the wild-type strain using Student's $t$ test analysis $(* * p<0.05)$; for abbreviations of strains, see Fig. 2

1999). Congo red binding is only observed following growth at $26^{\circ} \mathrm{C}$ and absence of the hmsHFRS locus does not affect virulence in mammalian bubonic plague models (Perry et al. 2004). While the relationship between mice challenged via peripheral routes of infection and the presence of the $\mathrm{Crb}^{+}$phenotype is clear, no such relationship has been described for animals challenged via the intranasal route resulting in the pneumonic model of plague.

Our data indicate that $\mathrm{pCD} 1$ as well as $\mathrm{HmsF}$ are essential for virulence in the pneumonic plague model; the absence of one or more of these loci resulted in $Y$. pestis strains with decreased virulence. The precise role in virulence for pMT1 and pPCP1 is still unknown and no strain was available lacking only these two loci. When mice were challenged with strains possessing a full complement of the loci examined, all resulted in clinical disease with concurrent manifestations of disease confirmed at postmortem examination. During the study they exhibited ruffled fur, significant weight loss and appeared to be moribund at $72 \mathrm{~h}$ post-infection. All mice that received one of these fully virulent strains also exhibited increased lung hemorrhage with concomitant lung mass increase when compared with control groups. The mice which received any of these five strains also had much higher numbers of bacteria within the lung, spleen and liver tissues when compared with either uninfected control groups or to animals which received a mutant strain.

Animals challenged with $\mathrm{HmsF}^{-}$strains of $Y$. pestis, however, were not completely free of clinical symptoms of disease. These animals initially exhibited symptoms of disease, initially having ruffled fur and exhibiting body mass loss. These animals appeared to rapidly control the infection and by $48 \mathrm{~h}$ post-challenge, regained body mass and were not exhibiting signs of distress (no ruffled fur). These data indicate that, although initial infection appears to proceed at a similar rate to that with wild-type strains, the animals are able to overcome the infection relatively rapidly; this may be attributable to the loss of the HmsF phenotype and hence to the inability of these particular strains to sequester iron within the mouse macrophages.

The role for hemin- or CR-binding systems in host cell adherence, invasion and survival has been described for other pathogens (Daskaleros and Payne 1987; Garduno and Kay 1992; Stugard et al. 1989). Due to its ability to bind nitric oxide (NO), hemin can protect $\mathrm{HmsF}^{+} Y$. pestis cells from its bactericidal effects (Morris et al. 1995; VazquezTorres et al. 2008). This thesis is reinforced by results of Lillard et al. (1999), who described that the addition of hemin to $\mathrm{HmsF}^{+}$cells can led to an increased protection against the NO donor NOR-1. Thus in Y. pestis strains

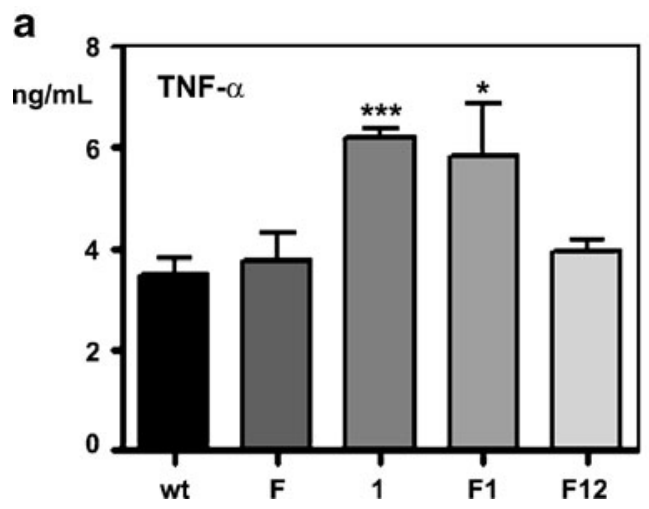

Fig. 5 TNF- $\alpha$ and IL-6 production (both in nanograms per milliliter) is altered by pCD1. Human PBMCs were infected with wild-type or mutant $Y$. pestis at a multiplicity of infection of 10 . At 4 and $48 \mathrm{~h}$ postinfection, respectively, TNF- $\alpha$ (a) and IL-6 (b) culture supernatant levels were determined by ELISA. Data are representative of three b

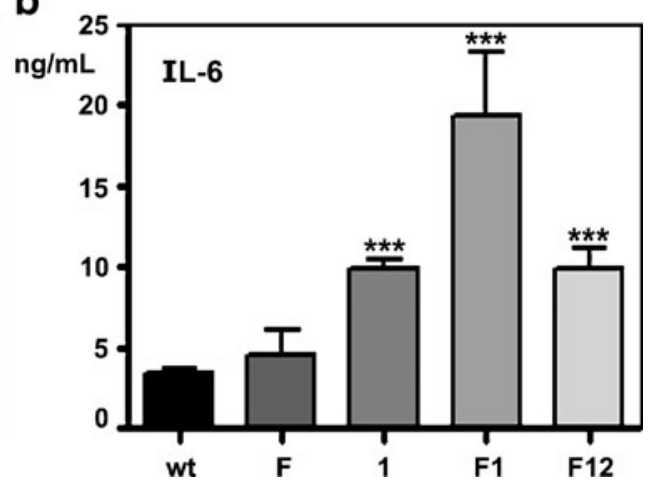

independent experiments; results are expressed as means \pm SEM. Significance was determined by comparison of the mutant strains to the wild-type strain using Student's $t$ test analysis $\left({ }^{*} p<0.05 ;{ }^{* * *} p<\right.$ 0.001); for abbreviations of strains, see Fig. 2 
lacking $\mathrm{HmsF}$ the antimicrobial function of $\mathrm{NO}$ is not inhibited by hemin. This may be an explanation for the increased bacterial clearance that was observed following infection with the $\mathrm{HmsF}^{-}$strains compared with the wildtype $Y$. pestis. Moreover, no changes in cytokine levels were detected after infection with $\mathrm{HmsF}^{-} Y$. pestis strains when compared with the wild-type strains. In contrast to the pCD1 plasmid, HmsF seems to have no function in the inhibition of cytokine production.

To survive inside the host and maintain a persistent infection, $Y$. pestis uses a variety of mechanisms to evade the host immune response ( $\mathrm{Li}$ and Yang 2008). One of those mechanisms is encoded by the pCD1 plasmid, namely the Type III Secretion System (TTSS) (Viboud and Bliska 2005). Y. pestis uses the TTSS to inject the Yersinia outer proteins (Yops) into macrophages, dendritic cells, and neutrophils to inhibit the responses of the host immune system (Cornelis 2002; Juris et al. 2002; Navarro et al. 2005). One of the key players in the TTSS is the pCD1 encoded LcrV protein (Smiley 2008). Besides its prominent role in delivering the Yops into the cell, LcrV is exposed on the bacterial cell surface to play a role in cell-cell adhesion (Holmstrom et al. 1997). After being secreted into the environment, LcrV exploits TLR2 and CD14 to trigger the release of IL-10 by the host immune cells to suppress the production of pro-inflammatory cytokines, such as TNF- $\alpha$ and IFN- $\gamma$ (Sing et al. 2003). This important role for pCD1 (or more specifically the TTSS), in inhibiting the host immune response and inducing cytotoxicity is confirmed by our results. PBMCs exposed to Y. pestis strains lacking the pCD1 plasmid, and thus the TTSS, showed increased cytokine levels and a slight decrease in cytotoxicity compared with the wild-type strains. The only exception is the $Y$. pestis strain which, besides pCD1, also lacks the HmsF and pMT1 markers; no increase in TNF- $\alpha$ levels was observed. Probably this unaltered TNF- $\alpha$ production is due to the absence of the Fraction 1 (F1) protein which is encoded by the pMT1 plasmid. DCs stimulated with Salmonella typhi-expressing F1 strain primed strong Agspecific CD4+ and CD8+ T cells (Ramirez et al. 2009). These cells secrete phagocyte-activating cytokines, such as IFN- $\gamma$ and TNF- $\alpha$ (Kaufmann 1993). This role for F1 in TNF- $\alpha$ production was confirmed by Sodhi et al. (2004) who showed that macrophages stimulated with $\mathrm{rF} 1$ for $24 \mathrm{~h}$ produce high TNF- $\alpha$ levels. The absence of both the cytokine suppressing $\mathrm{pCD} 1$ as well as the cytokine stimulating pMT1 may be the explanation of the unaltered TNF- $\alpha$ levels that we observed in vitro. However, after infection with strains lacking pCD1, HmsF and pMT1, a significant increase in IL-6 level was observed. This suggests that the pMT1 plasmid (probably its product the F1 antigen), stimulates the production of TNF- $\alpha$ without affecting the expression of the IL-6 cytokine. The precise function of the pMT1 plasmid in the above process remains unknown as no $Y$. pestis strain lacking the pMT1 plasmid was available during this study. Clearly, this comparative in vivo virulence study provides novel insights into the role of $\mathrm{HmsF}$ in pneumonic plague.

Open Access This article is distributed under the terms of the Creative Commons Attribution Noncommercial License which permits any noncommercial use, distribution, and reproduction in any medium, provided the original author(s) and source are credited.

\section{References}

Agar SL, Sha J, Foltz SM, Erova TE, Walberg KG, Parham TE, Baze WB, Suarez G, Peterson JW, Chopra AK (2008) Characterization of a mouse model of plague after aerosolization of Yersinia pestis CO92. Microbiology 154:1939-1948

Agar SL, Sha J, Foltz SM, Erova TE, Walberg KG, Baze WB, Suarez G, Peterson JW, Chopra AK (2009) Characterization of the rat pneumonic plague model: infection kinetics following aerosolization of Yersinia pestis CO92. Microbes Infect 11:205-214

Anderson DM, Ciletti NA, Lee-Lewis H, Elli D, Segal J, DeBord KL, Overheim KA, Tretiakova M, Brubaker RR, Schneewind O (2009) Pneumonic plague pathogenesis and immunity in Brown Norway rats. Am J Pathol 174:910-921

Brubaker RR (1969) Mutation rate to nonpigmentation in Pasteurella pestis. J Bacteriol 98:1404-1406

Cornelis GR (2002) Yersinia type III secretion: send in the effectors. J Cell Biol 158:401-408

Daskaleros PA, Payne SM (1987) Congo red binding phenotype is associated with hemin binding and increased infectivity of Shigella flexneri in the HeLa cell model. Infect Immun 55:1393-1398

Garduno RA, Kay WW (1992) Interaction of the fish pathogen Aeromonas salmonicida with rainbow trout macrophages. Infect Immun 60:4612-4620

Hare JM, McDonough KA (1999) High-frequency RecA-dependent and -independent mechanisms of Congo red binding mutations in Yersinia pestis. J Bacteriol 181:4896-4904

Holmstrom A, Petterson J, Rosqvist R, Hakansson S, Tafazoli F, Fallman M, Magnusson KE, Wolf-Watz H, Forsberg A (1997) YopK of Yersinia pseudotuberculosis controls translocation of Yop effectors across the eukaryotic cell membrane. Mol Microbiol 24:73-91

Juris SJ, Shao F, Dixon JE (2002) Yersinia effectors target mammalian signalling pathways. Cell Microbiol 4:201-211

Kaufmann SH (1993) Immunity to intracellular bacteria. Ann Rev Immunol 11:129-163

Li B, Yang R (2008) Interaction between Yersinia pestis and the host immune system. Infect Immun 76:1804-1811

Lillard JW Jr, Bearden SW, Fetherston JD, Perry RD (1999) The hemin storage $\left(\mathrm{Hms}^{+}\right)$phenotype of Yersinia pestis is not essential for the pathogenesis of bubonic plague in mammals. Microbiology 145(Part 1):197-209

Matero P, Pasanen T, Laukkanen R, Tissari P, Tarkka E, Vaara M, Skurnik M (2009) Real-time multiplex PCR assay for detection of Yersinia pestis and Yersinia pseudotuberculosis. APMIS 117:34-44

Morris CJ, Earl JR, Trenam CW, Blake DR (1995) Reactive oxygen species and iron - a dangerous partnership in inflammation. Int $\mathrm{J}$ Biochem Cell Biol 27:109-122

Navarro L, Alto NM, Dixon JE (2005) Functions of the Yersinia effector proteins in inhibiting host immune responses. Curr Opin Microbiol 8:21-27 
Perry RD, Fetherston JD (1997) Yersinia pestis - etiologic agent of plague. Clin Microbiol Rev 10:35-66

Perry RD, Pendrak ML, Schuetz P (1990) Identification and cloning of a hemin storage locus involved in the pigmentation phenotype of Yersinia pestis. J Bacteriol 172:5929-5937

Perry RD, Bobrov AG, Kirillina O, Jones HA, Pedersen L, Abney J, Fetherston JD (2004) Temperature regulation of the hemin storage $\left(\mathrm{Hms}^{+}\right)$phenotype of Yersinia pestis is posttranscriptional. J Bacteriol 186:1638-1647

Prentice MB, Rahalison L (2007) Plague. Lancet 369:1196-1207

Ramirez K, Capozzo AV, Lloyd SA, Sztein MB, Nataro JP, Pasetti MF (2009) Mucosally delivered Salmonella typhi expressing the Yersinia pestis $\mathrm{F} 1$ antigen elicits mucosal and systemic immunity early in life and primes the neonatal immune system for a vigorous anamnestic response to parenteral F1 boost. J Immunol 182:1211-1222

Sing A, Tvardovskaia N, Rost D, Kirschning CJ, Wagner H, Heesemann J (2003) Contribution of toll-like receptors 2 and 4 in an oral Yersinia enterocolitica mouse infection model. Int $\mathrm{J}$ Med Microbiol 293:341-348

Smiley ST (2008) Immune defense against pneumonic plague. Immunol Rev 225:256-271

Sodhi A, Sharma RK, Batra HV, Tuteja U (2004) Recombinant fraction 1 protein of Yersinia pestis activates murine peritoneal macrophages in vitro. Cell Immunol 229:52-61
Staggs TM, Fetherston JD, Perry RD (1994) Pleiotropic effects of a Yersinia pestis fur mutation. J Bacteriol 176:7614-7624

Stugard CE, Daskaleros PA, Payne SM (1989) A 101-kilodalton heme-binding protein associated with congo red binding and virulence of Shigella flexneri and enteroinvasive Escherichia coli strains. Infect Immun 57:3534-3539

Tomaso H, Jacob D, Eickhoff M, Scholz HC, Al Dahouk S, Kattar MM, Reischl U, Plicka H, Olsen JS, Nikkari S, Matero P, Beuret C, Ciammaruconi A, Lista F, Gala JL, Broll H, Appel B, Sellek Cano RE, Ybarra D, Broekhuijsen M, Indra A, Petersen R, Neubauer H (2008) Preliminary validation of real-time PCR assays for the identification of Yersinia pestis. Clin Chem Lab Med 6:1239-1244

Vazquez-Torres A, Stevanin T, Jones-Carson J, Castor M, Read RC, Fang FC (2008) Analysis of nitric oxide-dependent antimicrobial actions in macrophages and mice. Methods Enzymol 437:521-538

Viboud GI, Bliska JB (2005) Yersinia outer proteins: role in modulation of host cell signaling responses and pathogenesis. Ann Rev Microbiol 59:69-89

Zauberman A, Tidhar A, Levy Y, Bar-Haim E, Halperin G, Flashner Y, Cohen S, Shafferman A, Mamroud E (2009) Yersinia pestis endowed with increased cytotoxicity is avirulent in a bubonic plague model and induces rapid protection against pneumonic plague. PLoS One 4:e5938 